\title{
Conjugate flows and amplitude bounds for internal solitary waves
}

\author{
N. I. Makarenko ${ }^{1,2}$, J. L. Maltseva ${ }^{1,2}$, and A. Yu. Kazakov ${ }^{1}$ \\ ${ }^{1}$ Lavrentyev Institute of Hydrodynamics 630090, Novosibirsk, Russia \\ ${ }^{2}$ Novosibirsk State University 630090, Novosibirsk, Russia
}

Received: 4 November 2008 - Revised: 19 February 2009 - Accepted: 19 February 2009 - Published: 4 March 2009

\begin{abstract}
Amplitude bounds imposed by the conservation of mass, momentum and energy for strongly nonlinear waves in stratified fluid are considered. We discuss the theoretical scheme which allows to determine broadening limits for solitary waves in the terms of a given upstream density profile. Attention is focused on the continuously stratified flows having multiple broadening limits. The role of the mean density profile and the influence of fine-scale stratification are analyzed.
\end{abstract}

\section{Introduction}

The topic includes fundamental aspects of the wave propagation. In the present paper, we discuss the limitations for a steady internal waves caused by the integrals of the motion. According to Amick and Turner (1986), two different limit cases are possible for extreme solitary waves. In the first case, one deals with broad waves having flat plateau-shaped crests and almost parallel midsection flow. In the second case, the overhanging occurs for mushroom-shaped wave profiles possessing vertical tangents on steep fronts. The overhanging waves have been calculated numerically for exact fully nonlinear Euler equations by Holyer (1979), Saffman and Yuen (1982), Meiron and Saffman (1983), and Rusås and Grue (2002). Funakoshi and Oikawa (1986), Turner and Vanden-Broeck (1988) and Evans and Ford (1996) have confirmed also numerically the existence of plateau-shaped solitary waves in a two-layer fluid. These waves have the fronts being similar to the smooth bores when the midsection flow becomes a horizontal flow conjugated with far upstream. The pair of 1D stratified flows are conjugated if they possess the same mass,

Correspondence to: N. I. Makarenko (makarenko@hydro.nsc.ru) momentum and (or) energy fluxes. Benjamin $(1966,1971)$ formulated and investigated the problem on conjugate flows in order to find matching conditions for a non-uniform upand down-stream over topography. Here we consider internal solitary waves travelling over flat bottom. It is known in this case that the wave amplitude of extreme interfacial solitary wave does not exceed the critical value which may be found a priori as the amplitude of dissipationless bore. Weakly nonlinear theory of long waves describes this effect in the framework of extended $\mathrm{KdV}$ equation with a cubic nonlinearity (see the survey by Helfrich and Melville, 2006).

It should be noted that both limit forms of solitary waves can attend simultaneously. Grimshaw and Pullin (1986), Turner and Vanden-Broeck (1986) calculated the families of permanent waves having multiple returns from the broadening to the overhanging by the variation of control parameters. Moreover, Dias and Vanden-Broeck (2003) computed directly interfacial smooth bore of large amplitude having overhanging front. However, Rusås and Grue (2002) demonstrated numerically that the elevation of overhanging waves are considerably smaller than those predicted by the conjugate flow theory.

Overturning of extreme waves motivates one to consider theoretical schemes for a description of fluid motion prior to the breaking and much later after it. Breaking of strongly nonlinear internal waves can occur not only at the steep fronts but also near the middle of broad wave crests, as it was demonstrated experimentally by Grue et al. (2000). Using the matched expansion technique, Derzho and Grimshaw (1997) considered solitary waves with a recirculation zone formed over flat crests after the attainment of limit amplitude. The middle flow at the center of this wave can be modelled by conjugate flow theory with trapped core (Lamb and Wilkie, 2004). Recently, Scotti and Pineda (2005) observed internal waves which have attached vortex cores propagating over the slope of continental shelf. 
In the present paper, we consider regular solitary waves being close to the wave of limit amplitude. Maltseva (2003) discussed the form of a table-top solitary wave with amplitude which exceed the first limit value. Such a wave can appear only in the case when the wave amplitude has several critical values. This effect can occur when the midsection conjugate flow is not unique for a given upstream current. Non-uniqueness of conjugate flows was found numerically by Lamb and Wan (1998) for stratification with two pycnoclines and studied analytically by Makarenko (1999) for the density profile close to a linear or exponential one. Here we consider the case of a general continuous stratification in more details in order to characterize the role of vertical structure of the fluid density in the context of extreme wave forms.

\section{Governing equations}

Basic equations describing a two-dimensional motion of a heavy inviscid inhomogeneous fluid are as follows

$$
\begin{aligned}
& \rho\left(\frac{\partial U}{\partial t}+U \frac{\partial U}{\partial x}+V \frac{\partial U}{\partial y}\right)+\frac{\partial p}{\partial x}=0, \\
& \rho\left(\frac{\partial V}{\partial t}+U \frac{\partial V}{\partial x}+V \frac{\partial V}{\partial y}\right)+\frac{\partial p}{\partial y}=-\rho g, \\
& \frac{\partial \rho}{\partial t}+U \frac{\partial \rho}{\partial x}+V \frac{\partial \rho}{\partial y}=0, \\
& \frac{\partial U}{\partial x}+\frac{\partial V}{\partial y}=0
\end{aligned}
$$

where $\rho$ is the fluid density, $(U, V)$ is the fluid velocity, $p$ is the pressure and $g$ is the gravity acceleration. The flow domain is confined between flat bottom $y=0$ and rigid lid $y=h$ with the boundary condition

$V=\left.0\right|_{y=0, y=h}$.

We consider permanent waves travelling along the $x$-axes with constant phase speed $c$. In the frame moving with the wave, the flow becomes to be stationary. We introduce the stream function $\psi$ by means of $U=\psi_{y}, \quad V=-\psi_{x}$, so the Eq. (3) implies the dependence $\rho=\rho(\psi)$ for a steady flow. In addition, we involve the Bernoulli equation

$\frac{1}{2}|\nabla \psi|^{2}+\frac{p}{\rho(\psi)}+g y=\beta(\psi)$

in order to eliminate the pressure. Thus, the Eqs. (1)-(2) are reduced to the Dubreil-Jacotin -Long (DJL) equation

$\rho(\psi) \nabla^{2} \psi+\rho_{\psi}^{\prime}(\psi)\left(g y+\frac{1}{2}|\nabla \psi|^{2}\right)=H_{\psi}^{\prime}(\psi)$,

where $H(\psi)=\rho(\psi) \beta(\psi)$. The dependence $\rho$ and $\beta$ on $\psi$ can be specified by upstream condition for solitary wave solutions. Namely, we suppose that the flow tends to uniform flow defined by the stream function $\psi_{\infty}(y)=c y$ and known density profile $\rho_{\infty}(y)$ as $x \rightarrow-\infty$. Therefore, we obtain

$\rho(\psi)=\rho_{\infty}(\psi / c)$,

$H_{\psi}^{\prime}(\psi)=\rho_{\psi}^{\prime}(\psi)\left(\frac{g \psi}{c}+\frac{1}{2} c^{2}\right)$.

In terms of the stream function, boundary condition Eq. (5) takes the form

$\psi=\left.0\right|_{y=0}, \quad \psi=\left.c h\right|_{y=h}$.

Conjugate flow theory suggested in the context of broadening effect for solitary waves uses the conservation of total mass, energy and momentum of fluid layer. The derivation procedure of the DJL Eq. (7) takes into account automatically the conservation of mass flux by fixing dependence $\rho(\psi)$. The same arguments using fixed Bernoulli function $\beta(\psi)$ provide conservation of total energy. In contrast, momentum equation gives non-trivial compatibility condition $F=F_{\infty}$ where $F$ is the flow force integral (horizontal momentum flux),

$F=\int_{0}^{h}\left(p+\rho \psi_{y}^{2}\right) d y$.

Basic dimensionless parameters for a continuously stratified flow are the Boussinesq parameter $\sigma$ and the inverse densimetric Froude number $\lambda$,

$\sigma=\frac{N_{0}^{2} h}{\pi g}, \quad \lambda=\frac{\sigma g h}{\pi c^{2}}$,

where $N_{0}$ is the typical value of the Brunt-Väisälä frequency, $N^{2}(y)=-g \rho_{\infty}^{\prime}(y) / \rho_{\infty}(y)$. We scale the denominator in familiar expressions for parameters $\sigma$ and $\lambda$ by the multiplier $\pi=3.14 \ldots$ bearing in mind trigonometric modal functions which appear in the case of linear or exponential stratification. Non-dimensional form of the DJL equation uses dimensionless variables

$(x, y)=\frac{h}{\pi}(\bar{x}, \bar{y}), \quad \psi=\frac{c h}{\pi} \bar{\psi}$.

Dropping the bar we obtain from Eqs. (7) and (10) the equations for deviation from uniform flow $v(x, y)=\psi(x, y)-y$,

$$
\begin{aligned}
& \frac{\partial}{\partial x}\left\{\rho(y+v) \frac{\partial v}{\partial x}\right\}+\frac{\partial}{\partial y}\left\{\rho(y+v) \frac{\partial v}{\partial y}\right\}= \\
& =\rho_{\psi}^{\prime}(y+v)\left\{\sigma^{-1} \lambda v+\frac{1}{2}\left(\frac{\partial v}{\partial x}\right)^{2}+\frac{1}{2}\left(\frac{\partial v}{\partial y}\right)^{2}\right\}, \\
& v=\left.0\right|_{y=0, y=\pi} .
\end{aligned}
$$

Excluding the pressure $p$ from Eq. (11), we rewrite the momentum equation in accordance with variational 
formulation given by Benjamin (1984), so the flow force integral can be presented in dimensionless form as

$$
\int_{0}^{\pi}\left\{\rho(y+v)\left(\frac{\partial v}{\partial x}\right)^{2}+L\right\} d y=0
$$

where $L$ is the Lagrangian of the DJL equation,

$$
L=-\frac{1}{2} \rho(y+v)|\nabla v|^{2}+\sigma^{-1} \lambda \int_{y}^{\psi}(\rho(\chi)-\rho(\psi)) d \chi .
$$

\section{Interfacial waves in a two-fluid system}

In parallel with continuous stratification, we consider first two-layered irrotational flows with the interface $y=\eta(x)$ between the fluids having the constant densities $\rho_{2}$ in the upper layer and $\rho_{1}>\rho_{2}$ in the lower layer. For a steady fully nonlinear waves, we obtain instead of Eq. (7) the Laplace equation

$\nabla^{2} \psi=0$

for $0<y<\eta(x)$ and $\eta(x)<y<h$. The piece-wise uniform flow having $\eta(x)=h_{1}$ and the stream function

$\psi_{\infty}(y)= \begin{cases}c_{1} y, & \left(0<y<h_{1}\right), \\ c_{1} h_{1}+c_{2}\left(y-h_{1}\right), & \left(h_{1}<y<h\right)\end{cases}$

will be used as the limit flow at infinity: $\psi \rightarrow \psi_{\infty}(x \rightarrow-\infty)$. Here $c_{j}(j=1,2)$ is the wave speed with respect to the $j$ th layer. The stream function should satisfy the conditions at the rigid walls

$\psi=\left.0\right|_{y=0}, \quad \psi=c_{1} h_{1}+\left.c_{2} h_{2}\right|_{y=h}$

where $h=h_{1}+h_{2}$ is the total fluid depth. The boundary conditions at the interface $y=\eta(x)$ are

$\psi=c_{1} h_{1}, \quad[\psi]=0$,

$\left[\rho\left(\psi_{x}^{2}+\psi_{y}^{2}+2 g y-2 \beta\right)\right]=0$,

where $\beta$ is the Bernoulli constant, and square brackets denote the discontinuity jump. The two-layer flow force integral reduces to the form

$$
\begin{aligned}
& F=\rho_{1} \int_{0}^{\eta}\left(\psi_{y}^{2}-\psi_{x}^{2}\right) d y+\rho_{2} \int_{\eta}^{h}\left(\psi_{y}^{2}-\psi_{x}^{2}\right) d y- \\
& -\left(\rho_{1}-\rho_{2}\right) g \eta^{2}-2\left(\rho_{1} \beta_{1}-\rho_{2} \beta_{2}\right) \eta,
\end{aligned}
$$

where $\beta_{j}$ is the Bernoulli constant of the $j$ th layer. Far upstream condition for solitary waves gives

$$
\begin{aligned}
& F=\rho_{1} c_{1}^{2} h_{1}+\rho_{2} c_{2}^{2} h_{2}+g\left(\rho_{1}-\rho_{2}\right) h_{1}^{2}+2\left(\rho_{1} \beta_{1}-\rho_{2} \beta_{2}\right) h_{1}, \\
& 2 \beta_{1}=c_{1}^{2}+2 g h_{1}, \quad 2 \beta_{2}=c_{2}^{2}+2 g h_{1} .
\end{aligned}
$$

Basic dimensionless constants for a two-fluid system are the densimetric Froude numbers

$F_{j}^{2}=\frac{\rho_{j} c_{j}^{2}}{\left(\rho_{1}-\rho_{2}\right) g h} \quad(j=1,2)$

and the layer thickness ratio $r=h_{2} / h_{1}$. Linearized Eqs. (15)-(17) lead to the dispersion relation for a small sinusoidal waves with the wave-number $\varkappa$,

$F_{1}^{2} x \operatorname{coth} x+F_{2}^{2} x \operatorname{coth} r \varkappa=(1+r)^{-1}$.

This relation determines real wave-numbers if and only if the inequality

$F_{1}^{2}+r^{-1} F_{2}^{2} \leqslant(1+r)^{-1}$

is valid.

Steady non-linear long waves are described by the first-order ordinary differential equation obtained by Ovsyannikov (Ovsyannikov et al., 1985, Chpt. 1, §10, Eq. 10.6), by Miyata (1985), and also considered by Choi and Camassa (1999).

$\frac{1}{3}\left[\rho_{1} h_{1}^{2} c_{1}^{2}(h-\eta)+\rho_{2} h_{2}^{2} c_{2}^{2} \eta\right]\left(\frac{d \eta}{d x}\right)^{2}=$

$=-\eta(H-\eta)\left[\left(\rho_{1}-\rho_{2}\right) g \eta^{2}-2\left(\rho_{1} b_{1}-\rho_{2} b_{2}\right) \eta+F\right]+$

$+\rho_{1} h_{1}^{2} c_{1}^{2}(h-\eta)+\rho_{2} h_{2}^{2} c_{2}^{2} \eta$.

Using the dimensionless displacement $A(x)$ of interface introduced by $\eta(x)=h_{1}(1+A(x))$, we rewrite this ODE as follows

$$
\left(\frac{d A}{d x}\right)^{2}=3 A^{2} \frac{P(A)}{Q(A)}
$$

where

$$
\begin{aligned}
& P(A)= \\
& =F_{1}^{2}(r-A)+F_{2}^{2}(1+A)-(1+r)^{-1}(r-A)(1+A), \\
& Q(A)=F_{1}^{2}(r-A)+r^{2} F_{2}^{2}(1+A) .
\end{aligned}
$$

Solitary wave regimes are obtained depending on the multiplicity of the roots $a_{j}\left(F_{1}, F_{2}, r\right) \quad(j=1,2)$ of the numerator $P(A)$ on the right-hand side of Eq. (20). This polynomial $P$ should be positive for $-1<A<r$, and the positivity is valid if and only if the Froude numbers $F_{j}$ and the layer thickness ratio $r$ satisfy the inequality

$F_{1}^{2}+r^{-1} F_{2}^{2}>(1+r)^{-1}$.

The same inequality defines exterior domain with respect to the parametric set of small amplitude sinusoidal waves (see shadowed ellipse on Fig. 1). 


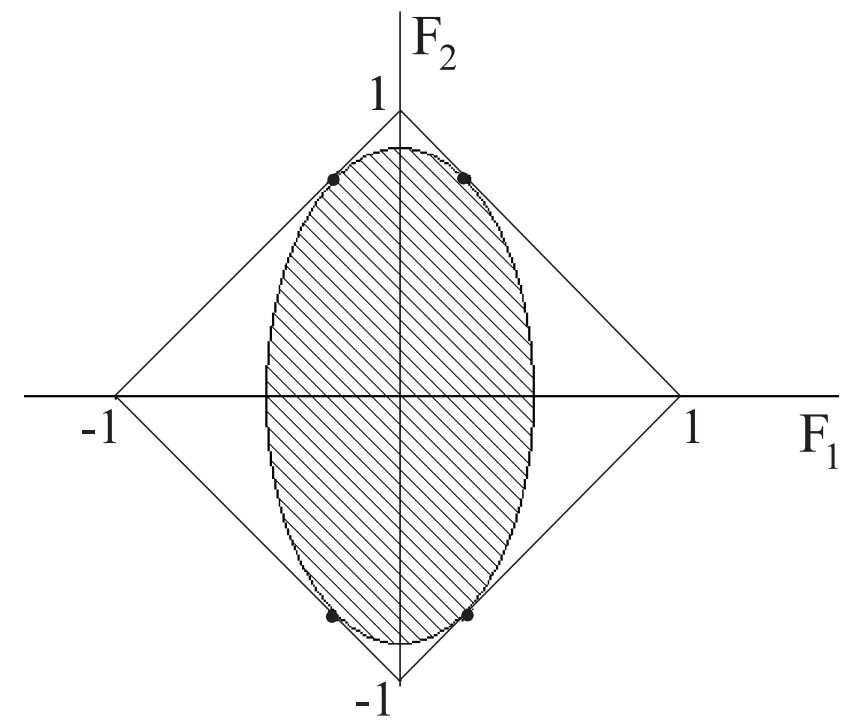

Fig. 1. Bore diagram for a two-layer fluid.

The bore solution of Eq. (20) corresponds to the double root $y=a$ which appears for the Froude numbers

$\left|F_{1}\right|=\frac{1+a}{1+r}, \quad\left|F_{2}\right|=\frac{r-a}{1+r}$.

Solitary-wave solutions of Eq. (20) exist for $\left(F_{1}, F_{2}\right)$ belonging to the supercritical domain Eq. (21) under the restriction $\left|F_{1}\right|+\left|F_{2}\right|<1$. The forms of a nonlinear waves can be obtained in explicit form if we replace approximately the denominator $Q(A)$ of Eq. (20) by the constant value $q_{*}=Q(0)$. Then the solitary wave is given by the formula

$A(x)=a \frac{1-\tanh ^{2} k x}{1-\theta^{2} \tanh ^{2} k x}, \quad k=\frac{a \sqrt{3 / q_{*}}}{2 \theta}$,

with $a=a_{1}$ and $\theta^{2}=a_{1} / a_{2}<1$, and the bore is given by

$A(x)=\frac{a}{2}(1+\tanh k x), \quad k=\frac{a \sqrt{3 / q_{*}}}{2}$.

The Froude numbers Eq. (22) also define all piece-wise constant flows which can be conjugated with another parallel flow. This fact follows immediately from the exact mass, momentum and energy relations. Existence of bore-like solution of Euler equations near a modified (cubic) KdV limit was proved rigorously in the paper by Makarenko (1992) for the Froude numbers Eq. (22) with sufficiently small $a$. Thus, the approximate Eq. (20) provides simple way to determine exact broadening limits for solitary waves in a two-layer fluid.

Functional equations for a three-layer conjugate flows are more complicated, these equation do not result an explicit analytical solution. However, Lamb (2000) and Rusås and Grue (2002) found numerically the non-uniqueness of critical amplitude for this case. Lamb and Wan (1998) found also numerically three branches of a fixed mode conjugate flows for a smooth density profile with two pycnoclines. In this paper, we discuss an interesting analogy between a continuous stratification (with smooth density profile $\rho_{\infty}(y)$ ) and both two-layer and three-layer cases.

\section{Continuous stratification}

Similarly to the case of a two-fluid system, we can reveal broad solitary waves using the parameters of limiting conjugate flow. It is supposed that all streamlines pass from $-\infty$ to $+\infty$ without formation of a recirculation zone. Therefore we obtain nonlinear eigenvalue problem for one-dimensional DJL equation

$\frac{d^{2} \psi}{d y^{2}}+\frac{\rho_{\psi}^{\prime}(\psi)}{\rho(\psi)}\left\{g y-\frac{g \psi}{c}+\frac{1}{2}\left(\frac{d \psi}{d y}\right)^{2}-\frac{1}{2} c^{2}\right\}=0$,

$\psi=\left.0\right|_{y=0}, \quad \psi=\left.c h\right|_{y=h}$,

where the wave velocity $c$ is a spectral parameter. The function $\psi(y)$ to be found should differ from the primary solution $\psi(y)=c y$. An additional scalar relation to Eqs. (23)-(24) appears due to the conservation of the momentum flux Eq. (11). Thus we have

$\int_{0}^{h} L d y=0$,

where $L$ is the Lagrangian of the one-dimensional DJL equation,

$L=-\frac{1}{2} \rho(\psi)\left\{\left(\frac{d \psi}{d y}\right)^{2}-c^{2}\right\}+g \int_{c y}^{\psi}(\rho(\chi)-\rho(\psi)) d \chi$.

Now we are looking for the flow conjugated with a weakly stratified constant current having the dimensionless density

$\rho(y)=1-\sigma \rho_{*}(y)-\sigma^{2} \rho_{1}(y, \sigma)$,

where the coefficient $\rho_{*}$ presents background density, and higher order term characterizes fine-scale stratification. There are few typical cases for a mean density stratification such as a linear or exponential dependence on depth, and also piece-wise constant density modelling homogeneous fluid layers with or without pycnoclines. Most real background profiles combine these simplest one. In contrast, fine vertical structure of the fluid density discovered for sea water by Kalle (1953) seems to be diverse, chaotic and variable. Figure 2 shows an example of the exponential-like mean profile and fine temperature stratification observed for ocean water by Fedorov (1978).

Equations (23)-(25) written for dimensionless deviation $v(y)=\psi(y)-y$ from primary flow, take the form 


$$
\begin{aligned}
& \frac{d}{d y}\left\{\rho(y+v) \frac{d v}{d y}\right\}= \\
& =\rho_{\psi}^{\prime}(y+v)\left\{\sigma^{-1} \lambda v+\frac{1}{2}\left(\frac{d v}{d y}\right)^{2}\right\}, \\
& v=\left.0\right|_{y=0, y=\pi}, \\
& \int_{0}^{\pi}\left\{-\frac{1}{2} \rho(y+v(y))\left(\frac{d v}{d y}\right)^{2}+\right. \\
& \left.+\sigma^{-1} \lambda \int_{y}^{y+v(y)}(\rho(\psi)-\rho(y+v(y))) d \psi\right\} d y=0 .
\end{aligned}
$$

Since we assume the parameter $\sigma$ to be small, we involve linearized Eqs. (27)-(28) written at the Boussinesq limit $\sigma=0$,

$\frac{d^{2} \phi}{d y^{2}}+\lambda \rho_{*}^{\prime}(y) \phi=0$,

$\phi=\left.0\right|_{y=0, y=\pi}$.

This problem determines normal modes with the eigenfunction $\left\{\phi_{n}\right\}_{n=1}^{\infty}$ corresponding to simple eigenvalues $\lambda_{n}$ $(n=1,2,3, \ldots)$. Using these eigenfunctions as the orthogonal basis, we are looking for 1-mode conjugate flow

$v(y)=b \phi_{1}(y)+b w(y ; \sigma, \lambda, b)$.

Here $b$ is amplitude parameter, and $w$ is the higher-order term being orthogonal to $\phi_{1}$. Substituting Eq. (32) into Eq. (27), we obtain

$\frac{d^{2} w}{d y^{2}}+\lambda_{1} \rho_{*}^{\prime}(y) w=G(w)$,

$w=\left.0\right|_{y=0, y=\pi}$,

where the nonlinearity $G(w)$ collects all terms from Eq. (27) beyond of linear part separated at the left-hand side of Eq. (33). This nonlinearity should satisfy the compatibility condition which has the form

$$
\begin{aligned}
& \int_{0}^{\pi} \phi_{1}(y)\left\{\frac{d}{d y}\left(\rho(\Psi(y)) \frac{d}{d y}\left(\phi_{1}(y)+w(y)\right)\right)-\right. \\
& -\rho_{\psi}^{\prime}(\Psi(y))\left(\sigma^{-1} \lambda\left(\phi_{1}(y)+w(y)\right)+\right. \\
& \left.\left.+\frac{b}{2}\left(\frac{d \phi_{1}(y)}{d y}+\frac{d w(y)}{d y}\right)^{2}\right)\right\} d y=0,
\end{aligned}
$$

where $\Psi(y)=y+b \phi_{1}(y)+b w(y ; \sigma, \lambda, b)$. In fact, the Eq. (35) defines scalar relation for $\lambda, \sigma, b$ such that Eq. (32)

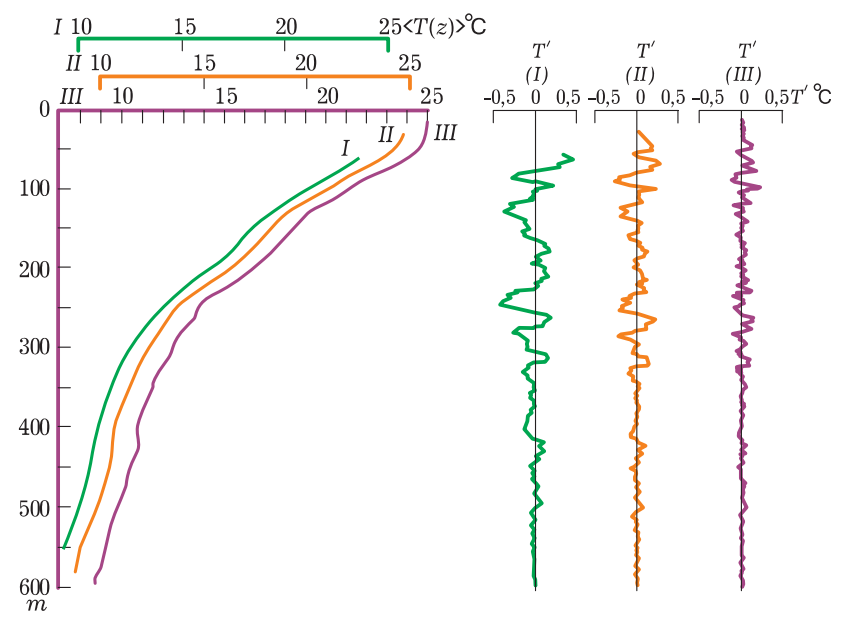

Fig. 2. Temperature stratification: mean profiles and fine-scale structure.

is a solution of Eqs. (27)-(28). Another scalar equation follows immediately from the flow force integral Eq. (29) after substitution Eq. (32),

$$
\begin{aligned}
& \int_{0}^{\pi}\left\{-\frac{1}{2} \rho(\Psi(y))\left(\frac{d \phi_{1}(y)}{d y}+\frac{d w(y)}{d y}\right)^{2}+\right. \\
& \left.+\frac{\lambda}{\sigma b^{2}} \int_{y}^{\Psi(y)}(\rho(\psi)-\rho(\Psi(y))) d \psi\right\} d y=0 .
\end{aligned}
$$

Trivial solution Eq. (32) with $b=0$ and arbitrary $\sigma, \lambda$ has been removed from Eqs. (35)-(36) by dividing with appropriate power of amplitude $b$. Note that last term of Eq. (36) is of finite order as $\sigma \rightarrow 0$ and $b \rightarrow 0$ while

$$
\begin{aligned}
& \int_{0}^{\pi} \int_{y}^{\Psi(y)}(\rho(\psi)-\rho(\Psi(y))) d \psi d y= \\
& =\frac{1}{2} \sigma b^{2} \int_{0}^{\pi} \frac{d \rho_{*}(y)}{d y}\left(\phi_{1}(y)+w_{0}(y)\right)^{2} d y+O\left(\sigma b^{3}\right)
\end{aligned}
$$

with a leading-order term $w_{0}(y)=w(y ; \sigma, \lambda, 0)$ (Appendix A). Therefore the system Eqs. (35), (36) can be presented as

$$
\begin{aligned}
& F_{0}(\lambda, \sigma)+b F_{1}(\lambda, \sigma)+O\left(b^{2}\right)=0, \\
& L_{0}(\lambda, \sigma)+b L_{1}(\lambda, \sigma)+O\left(b^{2}\right)=0 .
\end{aligned}
$$

As $\sigma$ is physical parameter one may control, we seek for a solution $\lambda=\lambda(\sigma), b=b(\sigma)$ satisfying the conditions $\lambda(0)=\lambda_{1}$ and $b(0)=0$. It is important that the Eqs. (38)-(39) still 


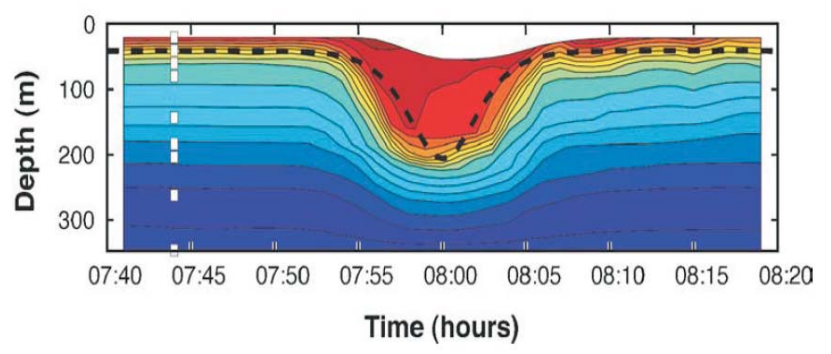

Fig. 3. Large amplitude internal solitary wave (from Duda et al., 2004; see also Helfrich and Melville, 2006). The dashed line is the profile of a KdV solitary wave calculated using the background stratification.

have the solution with amplitude $b(\sigma)=0$, vanishing by special choice $\lambda(\sigma)$. To demonstrate it, we set $b=0$ into Eqs. (38)-(39). By this way, we find

$$
\begin{aligned}
& F_{0}(\lambda, \sigma)=\int_{0}^{\pi} \phi_{1}(y)\left\{\frac{d}{d y}\left(\rho(y) \frac{d}{d y}\left(\phi_{1}(y)+w_{0}(y)\right)\right)-\right. \\
& \left.-\sigma^{-1} \lambda \frac{d \rho(y)}{d y}\left(\phi_{1}(y)+w_{0}(y)\right)\right\} d y .
\end{aligned}
$$

Similarly, integrate the Eq. (36) by parts, we obtain at leading order in $b$

$$
\begin{aligned}
& L_{0}(\lambda, \sigma)= \\
& =\frac{1}{2} \int_{0}^{\pi}\left(\phi_{1}(y)+w_{0}(y)\right)\left\{\frac{d}{d y}\left(\rho(y) \frac{d}{d y}\left(\phi_{1}(y)+w_{0}(y)\right)\right)-\right. \\
& \left.-\sigma^{-1} \lambda \frac{d \rho(y)}{d y}\left(\phi_{1}(y)+w_{0}(y)\right)\right\} d y
\end{aligned}
$$

At the same time, Eqs. (33)-(34) taken with $b=0$ have the form

$$
\begin{aligned}
& \frac{d}{d y}\left(\rho(y) \frac{d \Phi}{d y}\right)=\sigma^{-1} \lambda \frac{d \rho(y)}{d y} \Phi, \\
& \Phi=\left.0\right|_{y=0, y=\pi},
\end{aligned}
$$

where is denoted $\Phi(y)=\phi_{1}(y)+w_{0}(y)$. The Sturm-Liouville problem Eqs. (42)-(43) is a regular perturbation of Eqs. (30)-(30) by small parameter $\sigma$. This perturbed problem determines normal modes for a density profile Eq. (26) without Boussinesq approximation. Let $\left\{\tilde{\lambda}_{n}(\sigma)\right\}_{n=1}^{\infty}$ be perturbed eigenvalues. Since all these eigenvalues are simple, we have $\tilde{\lambda}_{1}(0)=\lambda_{1}$ where $\lambda_{1}$ is the lowest eigenvalue

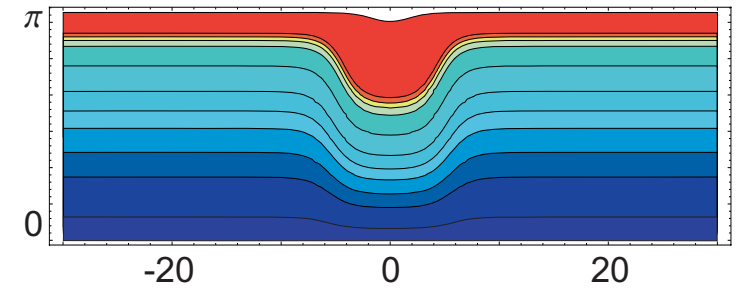

Fig. 4. Broad solitary wave given by the solution of Eq. (48) ( $b=0.9 ; \Lambda$ is the quadratic polynomial with a single minimum at $b_{0}=0.9025$.)

for the limiting problem Eqs. (30)-(30). Now it is easy to see from Eqs. (40)-(41) that both of coefficients $F_{0}(\lambda, \sigma)$ and $L_{0}(\lambda, \sigma)$ vanish by $\lambda=\tilde{\lambda}_{1}(\sigma)$. Hence we conclude that the system Eq. (38)-(39) has a special solution

$b(\sigma)=0, \lambda(\sigma)=\tilde{\lambda}_{1}(\sigma)$

This solution do not defines any non-trivial branch of conjugate flows, but it helps to specify the density profiles Eq. (26) which can admit this one. Let us rewrite the system Eqs. (38), (39) in the form

$\mathbf{A} q=f(q ; \sigma), \quad q=\left(b, \lambda-\lambda_{1}\right)$,

where the nonlinearity $f$ is of the order $O\left(|q|^{2}\right)$, and $\mathbf{A}$ is $2 \times 2$ matrix having the determinant (see Appendix B)

$$
\begin{aligned}
& \operatorname{det} A=\left.\left(L_{1} \frac{\partial F_{0}}{\partial \lambda}-F_{1} \frac{\partial L_{0}}{\partial \lambda}\right)\right|_{\lambda=\lambda_{1}, \sigma=0}= \\
& =\frac{\lambda_{1}}{6} \int_{0}^{\pi} \rho_{*}^{\prime}(y) \phi_{1}^{2}(y) d y \times \int_{0}^{\pi} \rho_{*}^{\prime \prime}(y) \phi_{1}^{3}(y) d y .
\end{aligned}
$$

Thus, if $\operatorname{det} \mathbf{A} \neq 0$ then the system Eqs. (35), (36) has only unique solution $\lambda=\lambda(\sigma), b=b(\sigma)$ such that $\lambda(0)=\lambda_{1}, b(0)=0$, and this solution is already found in Eq. (44). Therefore we can formulate the following condition.

Condition $\mathbf{A}$. The relation $\operatorname{det} \mathbf{A}=0$ is necessary to existence of 1-mode conjugate flows near the primary constant current having the density profile Eq. (26) with small $\sigma$.

\section{Linear density background}

The Condition A is obviously fulfilled for linear density $\rho=1-\sigma y$, as well as for an exponential stratification $\rho=\exp (-\sigma y)$. In this case, the Eq. (27) becomes linear within the weak stratification limit $\sigma=0$. Therefore the fine-scale density term of Eq. (26) is important by modelling nonlinear effects. The problem Eqs. (30)-(31) with $\rho_{*}(y)=y$ has the eigenfunctions and the eigenvalues

$\phi_{n}(y)=\sin n y, \quad \lambda_{n}=n^{2} \quad(n=1,2,3, \ldots)$. 


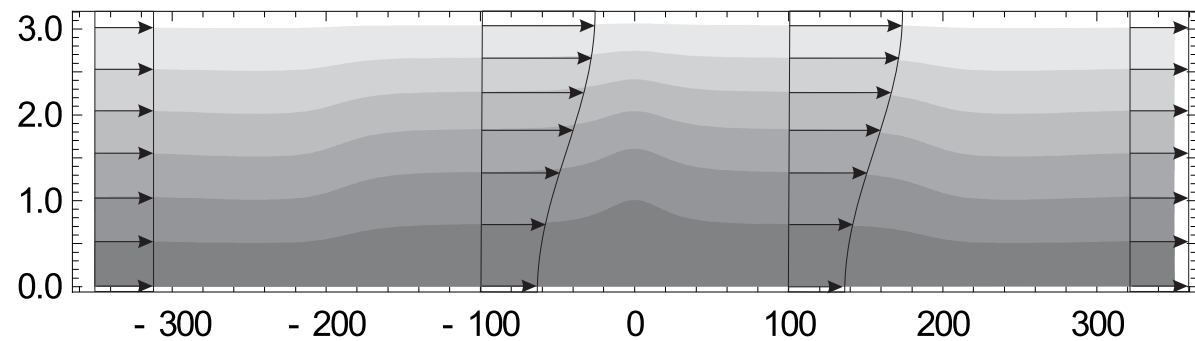

Fig. 5. The broad solitary waves with additional elevation in the midsection $(b=-0.596502, \sigma=1 / 20)$.

Now we transform the system of implicit scalar Eqs. (38)(38) for finite $b$ and small vector $q=\left(\sigma, \lambda-\lambda_{n}\right)$ to the more convenient form

$\mathbf{A}(b) q=f(q, b)$.

Here $\mathbf{A}$ is the Wronski matrix

$\mathbf{A}(b)=\left(\begin{array}{cc}s(b) & m(b) \\ s^{\prime}(b) & m^{\prime}(b)\end{array}\right)$

with the elements

$m(b)=\frac{1}{2} b^{2}$,

$s(b)=\frac{2}{\pi} \int_{0}^{\pi} \int_{y}^{y+b \sin y}\left(\rho_{0}(y+b \sin y)-\rho_{0}(\psi)\right) d \psi d y+$

$+\frac{\pi}{4} b^{2}+\frac{2}{3 \pi} b^{3}$

depending on the fine-scale stratification term $\rho_{0}(y)=\rho_{1}(y, 0)$. In the paper by Makarenko (1999), the sufficient existence condition was obtained for a conjugate flows of finite amplitude $|b|<1$. The bifurcation from primary flow occurs for the amplitude $b$ being close to the roots of the Wronskian

$\Delta(b)=-m^{2}(b) \frac{d}{d b}\left(\frac{s(b)}{m(b)}\right)$.

Condition B. Let $b_{0} \neq 0 \quad\left(\left|b_{0}\right|<1\right)$ be the simple root of the determinant $\Delta(b)$. Then for small $\sigma>0$ there exists the unique branch of conjugate flows such that $(b(\sigma), \lambda(\sigma))$ tends to $\left(b_{0}, 1\right)$ as $\sigma \rightarrow 0$.

The branch $\lambda(\sigma)$ of 1-mode conjugate flow corresponding to the root $b=b_{0}$ has the form

$\lambda(\sigma)=1-\frac{2 s\left(b_{0}\right)}{b_{0}^{2}} \sigma+O\left(\sigma^{2}\right)$.

So, the branches of conjugate flows are generated by the extreme points of the function

$\Lambda_{1}(b)=\frac{2 s(b)}{b^{2}}$

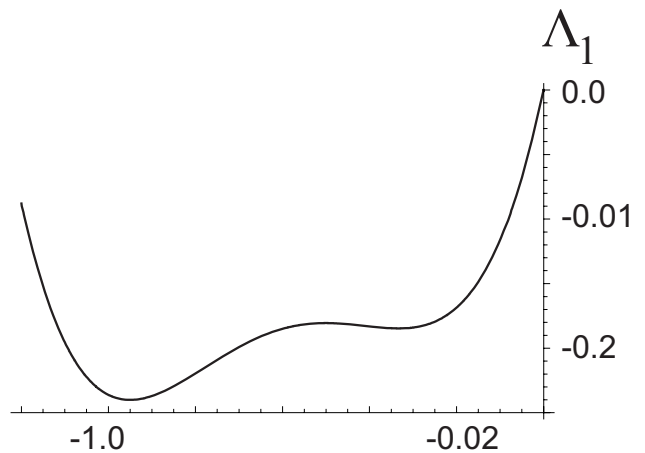

Fig. 6. The function $\Lambda_{1}$ for solitary wave shown in Fig. 5. Minima are at the points $b_{0}=-1 / 3, b_{1}=-19 / 20$.

The number of these extreme points $b_{0}$ depends on the fine-scale density coefficient $\rho_{0}$ only. The 1 -mode conjugate flow is supercritical with respect to the phase speed of infinitesimal waves while the inequality $\Lambda_{1}\left(b_{0}\right)<\Lambda_{1}(0)$ is satisfied. The minima give supercritical flows and the maxima provide subcritical flows. A possible alternate of subcritical and supercritical conjugate flows was conjectured first by Benjamin (1971) who used topological principles.

Further we consider first-mode plateau-shape solitary waves. We use the perturbation method suggested by Benney and Ko (1978) for large amplitude internal waves with linear stratification. We put

$\lambda=1+\sigma \Lambda_{1}(b)+O\left(\sigma^{2}\right)$

in Eq. (12) with amplitude $b$ bounded by $|b|<1$. In accordance with the Condition $\mathrm{B}$, the broadening values of $b$ are given by local maxima of the wave speed $c$ defined by the formula

$c^{2}=\frac{\sigma g h}{\pi\left(1+\sigma \Lambda_{1}(b)\right)}$

with the accuracy up to the order $O\left(\sigma^{3}\right)$. Looking for the power expansion

$v(x, y)=v_{0}(\xi, y)+\sigma v_{1}(\xi, y)+\ldots$ 


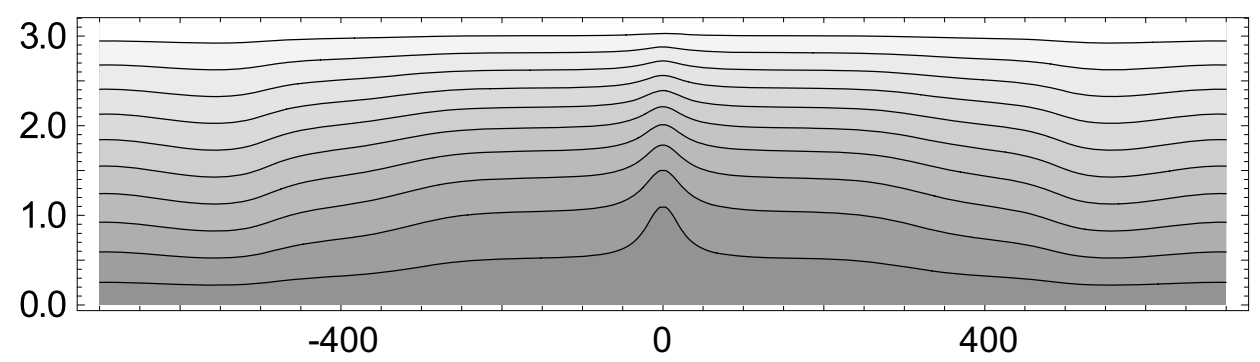

Fig. 7. The 2-level plateau-shape solitary wave with additional elevation in the midsection $(b=0.987105, \sigma=1 / 20)$.

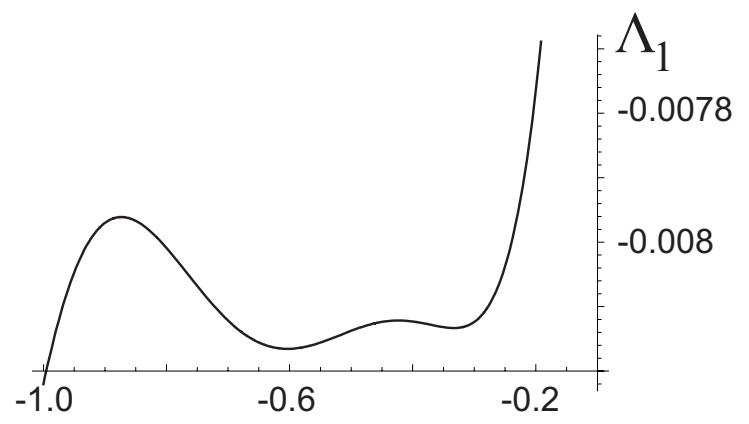

Fig. 8. The function $\Lambda_{1}$ for solitary wave shown in Fig. 7, minima are at $-1 / 3$ and -0.603333 , maxima are at -0.423333 and -0.873333 .

with slow independent variable $\xi=\sqrt{\sigma} x$, we obtain from Eq. (12)-(13) the set of equations

$v_{j y y}+v_{j}=f_{j}$,

$v_{j}=\left.0\right|_{y=0, y=\pi}$,

where $j=0,1, \ldots$ and $f_{0}=0$. The lowest-order solution has the form $v_{0}=B(x) \sin y$ where unknown function $B$ should satisfy vanishing condition of secular terms in the expression

$f_{1}=-v_{0 x x}+\left(y+v_{0}\right) v_{0 y y}-\rho_{0}^{\prime}\left(y+v_{0}\right) v+$

$+v_{0 y}+\frac{1}{2} v_{0 y}^{2}-\Lambda_{1}(b) v_{0}$.

Thus we obtain the equation

$$
\left(\frac{d B}{d \xi}\right)^{2}=B^{2}\left(\Lambda_{1}(B)-\Lambda_{1}(b)\right),
$$

which is similar to the Eq. (20) for interfacial solitary waves in a two-fluid system. Solitary wave solution exists for the amplitude $b$ such that $\Lambda_{1}^{\prime}(b) \neq 0$. The inequality $\Lambda_{1}(B)>\Lambda_{1}(b)$ means the supercriticality with respect to the propagation speed of infinitesimal waves. In accordance with the Condition B, the broadening limits for a fully nonlinear Euler equations are given by the minima of the function $\Lambda_{1}(b)$.
The Fig. 4 presents the broad solitary wave of depression in the case when the function $\Lambda_{1}$ has single minimum at the point $b_{0}$, and the amplitude $b$ is less than $b_{0}$. We choose the values of $b$ and $b_{0}$ so that the streamlines become similar to the temperature contour lines for solitary wave observed by Duda et al. (2004) (see Fig. 3). The Fig. 5 shows plateau-shape solitary wave having additional elevation at the flat crest. This elevation seems to be solitary wave propagating along with conjugate shear flow in the middle of the plateau. This is the case when the function $\Lambda_{1}(b)$ (see Fig. 6) generates four conjugate flows, and two of them are supercritical. Finally, Figs. 7 and 8 illustrate together one more exotic case when the solitary wave has two-level fronts and the elevation on the flat top.

\section{Conclusions}

In this paper, we have obtained the conditions which provide the existence of multiple broadening limits for internal solitary waves in a continuously stratified fluid. In accordance with this conditions, critical amplitude values are given by local maxima of the phase speed. The examples show that the wave speed depending on the fine-scale density can be non-monotone with respect to the wave amplitude, so the broadening limit can be not unique. The method used permits to consider large amplitude waves being far away from equilibrium state. This is because the perturbation scheme involves small density slope instead of small wave amplitude.

\section{Appendix A}

\section{Power expansion with respect to amplitude for the flow force integral}

Here we show that the integral

$$
I(b)=\int_{y}^{\Psi}(\rho(\psi)-\rho(\Psi)) d \psi
$$


N. I. Makarenko et al.: Conjugate flows and amplitude bounds for internal solitary waves

from Eq. (37) is of order $O\left(b^{2}\right)$ as $b \rightarrow 0$. By differentiating $I(b)$ we obtain

$\frac{\partial I(b)}{\partial b}=-b\left(\phi_{1}+w\right) \rho^{\prime}(\Psi) \frac{\partial \Psi}{\partial b}$.

Since this term is of order $O(b)$ and also $I(0)=0$, we have the power expansion

$I(b)=\frac{b^{2}}{2} \frac{\partial^{2} I(0)}{\partial b^{2}}+\frac{b^{3}}{6} \frac{\partial^{3} I(0)}{\partial b^{3}}+O\left(b^{4}\right)$.

From Eq. (A1) we obtain

$$
\begin{aligned}
& \frac{\partial^{2} I(b)}{\partial b^{2}}=-\rho^{\prime}(\Psi)\left(\frac{\partial \Psi}{\partial b}\right)^{2}- \\
& -b\left(\phi_{1}+w\right)\left(\rho^{\prime \prime}(\Psi)\left(\frac{\partial \Psi}{\partial b}\right)^{2}+\rho(\Psi) \frac{\partial^{2} \Psi}{\partial b^{2}}\right) .
\end{aligned}
$$

Taking into account that $\partial \Psi / \partial b=\phi_{1}+w_{0}$ at $b=0$, we find

$I(b)=-\frac{b^{2}}{2} \rho^{\prime}(y)\left(\phi_{1}+w_{0}\right)^{2}+O\left(b^{3}\right)$.

This formula implies Eq. (37) due to the decomposition Eq. (26) of the density $\rho$.

\section{Appendix B Determinant of the compatibility equations for conjugate flow problem}

Here we justify the formula Eq. (46) for the determinant of linear part of the system Eq. (45). From Eq. (40) we obtain

$$
\begin{aligned}
& \frac{\partial F_{0}\left(\lambda_{1}, 0\right)}{\partial \lambda}= \\
& =\int_{0}^{\pi} \phi_{1}\left(\frac{d^{2}}{d y^{2}} \frac{\partial w_{0}}{\partial \lambda}+\lambda_{1} \rho_{*}^{\prime}(y) \frac{\partial w_{0}}{\partial \lambda}+\rho_{*}^{\prime}(y) \phi_{1}\right) d y= \\
& =\int_{0}^{\pi} \rho_{*}^{\prime}(y) \phi_{1}^{2} d y .
\end{aligned}
$$

Terms containing $\partial w_{0} / \partial \lambda$ vanish after integration by parts since $\phi_{1}$ is an eigenfunction of Eq. (30).

Similarly taking into account that $w_{0}\left(y ; 0, \lambda_{1}\right)=0$ we find from Eq. (41)

$$
\begin{aligned}
& \frac{\partial L_{0}\left(\lambda_{1}, 0\right)}{\partial \lambda}= \\
& \frac{1}{2} \int_{0}^{\pi}\left\{\phi_{1}\left(\frac{d^{2}}{d y^{2}} \frac{\partial w_{0}}{\partial \lambda}+\lambda_{1} \rho_{*}^{\prime}(y) \frac{\partial w_{0}}{\partial \lambda}+\rho_{*}^{\prime}(y) \phi_{1}\right)+\right. \\
& \left.+\frac{\partial w_{0}}{\partial \lambda}\left(\frac{d^{2} \phi_{1}}{d y^{2}}+\lambda_{1} \rho_{*}^{\prime}(y) \phi_{1}\right)\right\} d y=
\end{aligned}
$$

$$
=\frac{1}{2} \int_{0}^{\pi} \rho_{*}^{\prime}(y) \phi_{1}^{2} d y .
$$

Then, differentiating Eq. (35) with respect to $b$ at $\sigma=0, \lambda=\lambda_{1}$ we obtain

$$
\begin{aligned}
& F_{1}\left(\lambda_{1}, 0\right)=\frac{d}{d b} \int_{0}^{\pi} \phi_{1}\left(\frac{d^{2} \phi_{1}}{d y^{2}}+\frac{d^{2} w}{d y^{2}}+\right. \\
& \left.+\lambda_{1} \rho_{*}^{\prime}(\Psi)\left(\phi_{1}+w\right)\right)\left.d y\right|_{b=0}= \\
& =\left.\int_{0}^{\pi} \phi_{1}\left\{\frac{d^{2}}{d y^{2}} \frac{\partial w}{\partial b}+\lambda_{1} \rho_{*}^{\prime}(y) \frac{\partial w}{\partial b}+\lambda_{1} \rho_{*}^{\prime \prime}(y) \phi_{1}^{2}\right\} d y\right|_{b=0}= \\
& =\lambda_{1} \int_{0}^{\pi} \rho_{*}^{\prime \prime}(y) \phi_{1}^{3} d y .
\end{aligned}
$$

Next, we compute $L_{1}\left(\lambda_{1}, 0\right)$. Using the expansion Eq. (A2) from Eq. (36) we obtain

$$
\begin{aligned}
& L_{1}\left(\lambda_{1}, 0\right)=\frac{\partial}{\partial b} \int_{0}^{\pi}\left\{-\frac{1}{2} \rho(\Psi)\left(\frac{d \phi_{1}}{d y}+\frac{d w}{d y}\right)^{2}+\right. \\
& \left.+\frac{\lambda_{1}}{\sigma b^{2}} \int_{y}^{\Psi}(\rho(\psi)-\rho(\Psi)) d \psi\right\}\left.d y\right|_{\sigma=0, b=0}= \\
& =\int_{0}^{\pi}\left\{-\left(\frac{d \phi_{1}}{d y}+\frac{d w}{d y}\right) \frac{d}{d y} \frac{\partial w}{\partial b}-\right. \\
& \left.-\frac{\lambda_{1}}{6} \frac{\partial^{3}}{\partial b^{3}} \int_{y}^{\Psi}\left(\rho_{*}(\psi)-\rho_{*}(\Psi)\right) d \psi\right\}\left.d y\right|_{b=0} .
\end{aligned}
$$

Then, taking into account that at $b=0, \sigma=0, \lambda=\lambda_{1}$

$w\left(y ; 0, \lambda_{1}, 0\right)=0, \quad \Psi=y$,

$\frac{\partial \Psi}{\partial b}=\phi_{1}, \quad \frac{\partial^{2} \Psi}{\partial b^{2}}=2 \frac{\partial w}{\partial b}$,

we obtain from Eq. (A3)

$$
\begin{aligned}
& \left.\frac{\partial^{3}}{\partial b^{3}} \int_{y}^{\Psi}\left(\rho_{*}(\psi)-\rho_{*}(\Psi)\right) d \psi\right|_{b=0}= \\
& -2 \rho_{*}^{\prime \prime}(y) \phi_{1}^{3}-6 \rho_{*}^{\prime}(y) \phi_{1} \frac{\partial w}{\partial b} .
\end{aligned}
$$

Nonlin. Processes Geophys., 16, 169-178, 2009 
Hence, using integration by parts we obtain from Eq. (B4)

$$
\begin{aligned}
& L_{1}\left(\lambda_{1}, 0\right)=\int_{0}^{\pi} \phi_{1}\left\{\frac{d^{2}}{d y^{2}} \frac{\partial w}{\partial b}+\lambda_{1} \rho_{*}^{\prime}(y) \frac{\partial w}{\partial b}+\right. \\
& \left.+\frac{\lambda_{1}}{3} \rho_{*}^{\prime \prime}(y) \phi_{1}^{2}\right\} d y=\frac{\lambda_{1}}{3} \int_{0}^{\pi} \rho_{*}^{\prime \prime}(y) \phi_{1}^{3} d y
\end{aligned}
$$

Finally, we find from Eqs. (B), (B2), (B3), (B5) that

$$
\begin{aligned}
& \operatorname{det} \mathbf{A}=F_{1}\left(\lambda_{1}, 0\right) \frac{\partial L_{0}}{\partial \lambda}\left(\lambda_{1}, 0\right)-L_{1}\left(\lambda_{1}, 0\right) \frac{\partial F_{0}}{\partial \lambda}\left(\lambda_{1}, 0\right)= \\
& =\frac{\lambda_{1}}{6} \int_{0}^{\pi} \rho_{*}^{\prime}(y) \phi_{1}^{2} d y \times \int_{0}^{\pi} \rho_{*}^{\prime \prime}(y) \phi_{1}^{3} d y
\end{aligned}
$$

which coincides with the Eq. (46).

Acknowledgements. This work was supported by the RFBR (grant No. 07-01-00309), INTAS-SB RAS (grant No. 06-9236), Russian Education Department (grant No. 2.1.1.4918) and Interdisciplinary Program of SB RAS (Project No. 113). NIM acknowledges also the support by RFBR-CNRS (grant No. 07-01-92212).

Edited by: R. Grimshaw

Reviewed by: one anonymous referee

\section{References}

Amick, C. and Turner, R. E. L.: A global theory of internal solitary waves in two-fluid system, Trans. Amer. Math. Soc., 298, 431484, 1986

Benjamin, T. B.: Internal waves of finite amplitude and permanent form, J.Fluid Mech., 25, 241-270, 1966.

Benjamin, T. B.: A unified theory of conjugate flows, Philos. Trans. Roy. Soc. London A., 269, 587-643, 1971.

Benjamin, T. B.: Impulse, flow force and variational principles, IMA J. Appl. Math., 32, 3-68, 1984.

Benney, D. J. and Ko, D. R. S.: The propagation of long large amplitude internal waves, Stud. Appl. Math., 59, 187-199, 1978.

Choi, W. and Camassa, R.: Fully nonlinear internal waves in a twofluid system, J.Fluid Mech., 396, 1-36, 1999.

Derzho, O. G. and Grimshaw, R.: Solitary waves with a vortex core in a shallow layer of stratified fluid, Phys. Fluids, 9, 3378-3385, 1997.

Dias, F. and Vanden-Broeck, J.-M.: On internal fronts, J. Fluid. Mech., 479, 145-154, 2003.

Duda, T. F., Lynch, J. F., Irish, J. D., Beardsley, J. D., Ramp, S. R., et al.: Internal tide and nonlinear wave behavior in the continental slope in the northern South China Sea, IEEE J. Ocean Eng., 29, 1105-1131, 2004.
Evans, W. A. B. and Ford, M. J.: An integral equation approach to internal (2-layer) solitary waves, Phys. Fluids, 8, 2032-2047, 1996.

Fedorov, K. N.: The thermohaline finestructure of the ocean, Pergamon marine series, Vol. 2. Pergamon, Oxford, 1978.

Funakoshi, M. and Oikawa, M.: Long internal waves of large amplitude in a two-layer fluid, J. Phys. Soc. Japan, 55, 128-144, 1986.

Grimshaw, R. H. L. and Pullin, D. I.: Extreme interfacial waves, Phys. of Fluids, 29, 2802-2807, 1986.

Grue, J., Jensen, A., Rusås, P.-O., and Sveen, J. K.: Breaking and broadening of internal solitary waves, J.Fluid Mech., 413, 181217, 2000.

Helfrich, K. R. and Melville, W. K.: Long nonlinear internal waves, Annu. Rev. Fluid Mech., 38, 395-425, 2006.

Holyer, J. Y.: Large amplitude progressive interfacial waves, J. Fluid Mech, 93, 433-448, 1979.

Kalle, K.: Zur Frage der inneren thermishe Unruhe des Meers, Deutsch. Hydrogr. Z., 6, 145-170, 1953.

Lamb, K. G. and Wan, B.: Conjugate flows and flat solitary waves for a continuously stratified fluid, Phys. Fluids, 10, 2061-2079, 1998.

Lamb, K. G.: Conjugate flows for a three-layer fluid, Phys. Fluids, 12, 2169-2185, 2000.

Lamb, K. G. and Wilkie, K.: Conjugate flows with trapped cores, Phys. Fluids, 16, 4685-4695, 2004.

Makarenko, N. I.: Smooth bore in a two-layer fluid, Int. Ser. Numerical Math. 106, 195-204, 1992.

Makarenko, N. I.: Conjugate flows and smooth bores in a weakly stratified fluid, J. Appl. Mech. Techn. Phys., 40, 249-257, 1999.

Maltseva, J. L.: Limiting forms of internal solitary waves, JOMAE Trans. ASME, 125, 76-79, 2003.

Meiron, D. I. and Saffman, P. G.: Overhanding interfacial gravity waves of large amplitude, J. Fluid Mech., 129, 213-218, 1983.

Mirie, R. M. and Pennel, S. A.: Internal solitary waves in a two-fluid system, Phys. Fluids A, 1, 986-991, 1989.

Miyata, M.: An internal solitary wave of large amplitude, La Mer, 23, 43-48, 1985.

Ovsyannikov, L. V., Makarenko, N. I., Nalimov, V. I., et al.: Nonlinear Problems of the Theory of Surface and Internal Waves, Nauka, Novosibirsk, 1985 (in Russian).

Rusås, P.-O. and Grue, J.: Solitary waves and conjugate flows in a three-layer fluid, Eur. J. Mech. B/Fluids, 21, 185-206, 2002.

Saffman, P. G. and Yuen, H. C.: Finite-amplitude interfacial waves in the presence of a current, J. Fluid Mech., 123, 459-476, 1982.

Scotti, A. and Pineda, J.: Observation of very large and steep internal waves of elevation near the Massachusets coast, Geophys. Res. Lett., 31, L22307, doi:10.1029/2004GL021052, 2004.

Turner, R. E. L. and Vanden-Broeck, J.-M.: Limiting configuration of interfacial solitary waves, Phys. Fluids, 29, 372-375, 1986.

Turner, R. E. L. and Vanden-Broeck, J.-M.: Broadening of interfacial solitary waves, Phys. Fluids, 31, 2486-2490, 1988.

Yih, C. S.: Stratified flows, Academic Press, N.-Y, 1980. 